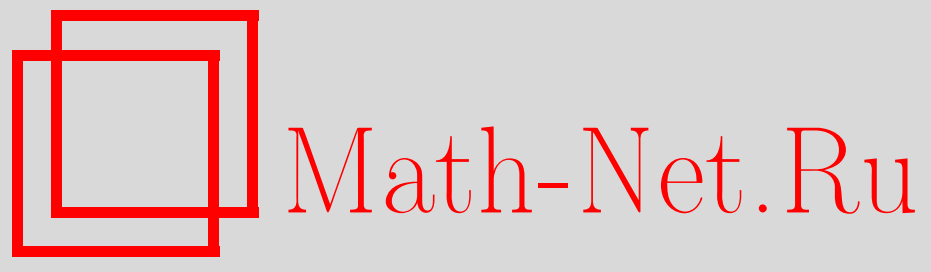

Ю. В. Малыхин, Оценки тригонометрических сумм по модулю $p^{r}$, Матем. заметки, 2006, том 80, выпуск $5,793-796$

DOI: https://doi.org/10.4213/mzm3092

Использование Общероссийского математического портала Math-Net.Ru подразумевает, что вы прочитали и согласны с пользовательским соглашением http://www . mathnet.ru/rus/agreement

Параметры загрузки:

IP : 18.209 .158 .208

26 апреля 2023 г., 13:24:18

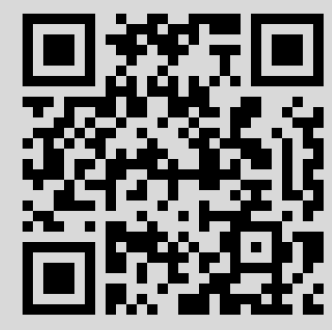




\section{ОЦЕНКИ ТРИГОНОМЕТРИЧЕСКИХ СУММ ПО МОДУЛЮ $p^{r}$}

\section{Ю. В. Малыхин}

Для натурального $q$ через $\mathbb{Z}_{q}$ мы будем обозначать кольцо вычетов по модулю $q$, а через $\mathbb{Z}_{q}^{*}$ - мультипликативную группу этого кольца. Для $x \in \mathbb{Z}_{q}$ обозначим $e_{q}(x):=$ $e^{2 \pi i x / q}$

Пусть $G$ - подгруппа $\mathbb{Z}_{q}^{*}$ порядка $t, a \in \mathbb{Z}_{q}$. Тригонометрическими суммами по подгруппе $G$ называются суммы вида $S(a, G):=\sum_{x \in G} e_{q}(a x)$. Наша задача будет состоять в оценке величины

$$
S(G):=\max _{a \in \mathbb{Z}_{q}^{*}}|S(a, G)|
$$

в случае, когда $q$ есть степень простого числа: $q=p^{r}$ (здесь и далее $p$ обозначает простое число, большее 2).

При достаточно больших $t$ оценки тригонометрических сумм по подгруппам $G \subset \mathbb{Z}_{q}^{*}$ основаны на оценке величин

$$
T_{k}(G):=\#\left\{\left(x_{1}, \ldots, x_{2 k}\right): x_{1}+\cdots+x_{k}=x_{k+1}+\cdots+x_{2 k}(\bmod q), x_{i} \in G\right\} .
$$

Имеет место

Лемма (основное неравенство). Для любых натуралъных $k$ ul

$$
S(G) \leqslant\left(q T_{k}(G) T_{l}(G)\right)^{1 /(2 k l)} t^{1-1 / k-1 / l} .
$$

Оценки такого типа установлены И.М. Виноградовым (метод “сглаживания") при оценках тригонометрических сумм Г. Вейля (см. [1]-[3]). Подобные неравенства содержатся также в [4]-[6]. Неравенство леммы является специальным вариантом упомянутых и доказано в книге С. В. Конягина и И. Е. Шпарлинского [7].

Рассмотрим простое $p$ и натуральное $r$. Можно показать, что если $G \subset \mathbb{Z}_{p^{r}}^{*}$ и $p$ $\# G$, то $S(G)=0$. Поэтому нам достаточно рассмотреть случай $\# G \mid p-1$. Итак, пусть $t \mid p-1$. Для каждого натурального $r$ существует единственная подгруппа $G_{r} \subset$ $\mathbb{Z}_{p^{r}}^{*}$ порядка $t$. Классический случай простого модуля, т.е. $r=1$, достаточно полно исследован. Современные оценки см. в [8], [9]. Случай $r=2$ рассмотрен в [10]. В данной работе мы получим оценки для величины $S\left(G_{r}\right)$ для всех $r \geqslant 3$ с помощью оценок для $T_{k}\left(G_{2}\right)$ и $S\left(G_{2}\right)$.

Teоpema 1. Пусть $T_{n}\left(G_{2}\right) \leqslant A p^{-2} t^{2 n}$. Тогда $n p u r \geqslant 3$

$$
S\left(G_{r}\right) \leqslant C(A, r) t p^{-\delta_{r, n}}, \quad \text { где } \quad \delta_{r, n}=\frac{1}{2 n^{2}(n+1)^{r-3}},
$$

для некоторой функиии $C$.

Число $n$ находится с помощью следующей теоремы.

Teорема 2. (i) Пусть $S\left(G_{1}\right) \leqslant S_{1}<t, S\left(G_{2}\right) \leqslant S_{2}<t, A>0$. Тогда при

$$
n=\max \left(1,\left\lceil\frac{1}{2} \frac{\ln (p t)-2 \ln S_{1}-\ln A}{\ln t-\ln S_{1}}\right\rceil,\left\lceil\frac{1}{2} \frac{\ln \left(p^{2} t\right)-2 \ln S_{2}-\ln A}{\ln t-\ln S_{2}}\right\rceil\right)
$$

выполняется неравенство $T_{n}\left(G_{2}\right) \leqslant(2 A+1) p^{-2} t^{2 n}$.

Работа выполнена при поддержке Российского фонда фундаментальных исследований, грант № 05-01-00066, и программы "Ведущие научные школы”, грант № НШ-3004.2003.1.

(C) Ю. В. МАлыхин, 2006 
(ii) Если дополнительно известно, что $T_{2}\left(G_{2}\right) \leqslant T$, то можно взять

$$
n=\max \left(2,\left\lceil\frac{1}{2} \frac{\ln (p t)-2 \ln S_{1}-\ln A}{\ln t-\ln S_{1}}\right\rceil,\left\lceil\frac{1}{2} \frac{\ln \left(p^{2} T\right)-4 \ln S_{2}-\ln A}{\ln t-\ln S_{2}}\right\rceil\right) .
$$

Из этих теорем вытекает

СледСтвие 1. При $(a, p)=1, r \geqslant 3$ имеем $\left|\sum_{x=1}^{p-1} e_{p^{r}}\left(a x^{p^{r-1}}\right)\right| \leqslant C(r) p^{1-1 /\left(32 \cdot 5^{r-3}\right)}$.

Для доказательства теорем нам потребуются несколько лемм.

Лемма 1. Если $r>s, \operatorname{mo~}_{k}\left(G_{r}\right) \leqslant T_{k}\left(G_{s}\right)$.

ДоказАтельство. Достаточно доказать лемму в случае $r=s+1$. Покажем, что гомоморфизм $\bmod p^{s}: \mathbb{Z}_{p^{s+1}} \rightarrow \mathbb{Z}_{p^{s}}$ переводит $G_{s+1}$ в $G_{s}$, притом взаимно однозначно. Пусть $d=(p-1) / t$. Тогда $G_{s+1}=\left\{x^{p^{s} d}: x \in \mathbb{Z}_{p^{s+1}}^{*}\right\}$. Поскольку $\forall a \in \mathbb{Z}_{p^{s}}^{*} a^{p^{s-1}(p-1)}=1$, для $(x, p)=1$ получаем

$$
x^{p^{s} d}=\left(x^{d}\right)^{p^{s}}=\left(x^{d}\right)^{p^{s-1}}=x^{p^{s-1} d}\left(\bmod p^{s}\right) .
$$

Теперь ясно, что $\bmod p^{s}$ переводит $G_{s+1}$ в $G_{s}$, причем сюрьективно, а, значит, биективно. Поэтому каждому решению уравнения $x_{1}+\cdots+x_{k}=x_{k+1}+\cdots+x_{2 k}\left(\bmod p^{s+1}\right)$ соответствует свое решение уравнения $x_{1}+\cdots+x_{k}=x_{k+1}+\cdots+x_{2 k}\left(\bmod p^{s}\right)$.

Лемма 2. Имеем

$$
\sum_{a \in \mathbb{Z}_{p^{r}}}\left|S\left(a, G_{r}\right)\right|^{l}=\sum_{a \in \mathbb{Z}_{p^{r}}^{*}}\left|S\left(a, G_{r}\right)\right|^{l}+\sum_{a \in \mathbb{Z}_{p^{r}-1}}\left|S\left(a, G_{r-1}\right)\right|^{l} .
$$

ДокАзАТЕЛЬСтво. Заметим, что $\mathbb{Z}_{p^{r}}=\mathbb{Z}_{p^{r}}^{*} \sqcup\left\{a \in \mathbb{Z}_{p^{r}}: p \mid a\right\}$. Когда $a$ в исходной сумме пробегает первое множество, получаем первое слагаемое. Второе множество можно отождествить с $\mathbb{Z}_{p^{r-1}}$ : если $p \mid a$, то положим $\varphi(a)=a / p$. Тогда $\varphi$ будет биекцией $\left\{a \in \mathbb{Z}_{p^{r}}: p \mid a\right\} \rightarrow \mathbb{Z}_{p^{r-1}}$. Теперь заметим, что $S\left(a, G_{r}\right)=S\left(\varphi(a), G_{r-1}\right)$, поэтому суммируя $\left|S\left(a, G_{r}\right)\right|^{l}$ по второму множеству, получаем второе слагаемое.

Теперь докажем основную теорему.

ДоКАЗАТЕЛЬСТВо ТЕОРЕмЫ 1 . Обозначим $k_{r}:=n(n+1)^{r-2}$.

Построим индукцией по $r$ такие функции $C_{1}(A, r)$ и $C_{2}(A, r)$, что

$$
S\left(G_{r}\right) \leqslant C_{1}(A, r) t p^{-1 /\left(2 k_{2} k_{r-1}\right)} \quad \text { при } r \geqslant 3
$$

и

$$
T_{k_{r}}\left(G_{r}\right) \leqslant C_{2}(A, r) p^{-r} t^{2 k_{r}} \quad \text { при } r \geqslant 2 .
$$

База индукции: при $r=2$ положим $C_{2}(A, 2)=A$.

Пусть мы построили функции $C_{1}$ и $C_{2}$ для всех номеров, меньших $r$. Построим их для $r$. Применяя основное неравенство при $q=p^{r}, k=k_{2}, l=k_{r-1}, G=G_{r}$, получим

$$
\begin{aligned}
S\left(G_{r}\right) & \leqslant\left(p^{r} A p^{-2} t^{2 k_{2}} C_{2}(A, r-1) p^{-r+1} t^{2 k_{r-1}}\right)^{1 /\left(2 k_{2} k_{r-1}\right)} t^{1-1 / k_{2}-1 / k_{r-1}} \\
& =\left\{A \cdot C_{2}(A, r-1)\right\}^{1 /\left(2 k_{2} k_{r-1}\right)} t p^{-1 /\left(2 k_{2} k_{r-1}\right)} .
\end{aligned}
$$

Полагая $C_{1}(A, r)=\left\{A \cdot C_{2}(A, r-1)\right\}^{1 /\left(2 k_{2} k_{r-1}\right)}$, получаем (4).

Теперь подберем $C_{2}(A, r)$ так, чтобы $p^{r} T_{k_{r}}\left(G_{r}\right) \leqslant C_{2}(A, r) p^{2 k_{r}}$; в силу леммы 2 имеем

$$
p^{r} T_{k_{r}}\left(G_{r}\right)=\sum_{a \in \mathbb{Z}_{p^{r}}}\left|S\left(a, G_{r}\right)\right|^{2 k_{r}}=\sum_{a \in \mathbb{Z}_{p^{*}}^{*}}\left|S\left(a, G_{r}\right)\right|^{2 k_{r}}+\sum_{a \in \mathbb{Z}_{p^{r}-1}}\left|S\left(a, G_{r-1}\right)\right|^{2 k_{r}} .
$$


Оценим второе слагаемое:

$$
\begin{aligned}
\sum_{a \in \mathbb{Z}_{p^{r}-1}}\left|S\left(a, G_{r-1}\right)\right|^{2 k_{r}} & \leqslant \sum_{a \in \mathbb{Z}_{p^{r}-1}}\left|S\left(a, G_{r-1}\right)\right|^{2 k_{r-1}} t^{2 k_{r}-2 k_{r-1}} \\
& =p^{1-r} T_{k_{r-1}}\left(G_{r-1}\right) t^{2 k_{r}-2 k_{r-1}} \leqslant C_{2}(A, r-1) t^{2 k_{r-1}} t^{2 k_{r}-2 k_{r-1}}
\end{aligned}
$$

по предположению индукции. Значит, второе слагаемое не превосходит $C_{2}(A, r-1) t^{2 k_{r}}$.

Оценим первое слагаемое:

$$
\begin{aligned}
\sum_{a \in \mathbb{Z}_{p^{*}}^{*}}\left|S\left(a, G_{r}\right)\right|^{2 k_{r}} & \leqslant \sum_{a \in \mathbb{Z}_{p^{r}}^{*}}\left|S\left(a, G_{r}\right)\right|^{2 k_{r-1}} \max _{a \in \mathbb{Z}_{p^{r}}^{*}}\left|S\left(a, G_{r}\right)\right|^{2 k_{r}-2 k_{r-1}} \\
& \leqslant p^{r} T_{k_{r-1}}\left(G_{r}\right) \cdot C_{1}(A, r)^{2 k_{r}-2 k_{r-1}} t^{2 k_{r}-2 k_{r-1}} p^{-\left(2 k_{r}-2 k_{r-1}\right) /\left(2 k_{2} k_{r-1}\right)} \\
& \leqslant p^{r} C_{2}(A, r-1) p^{-r+1} t^{2 k_{r-1}} C_{1}(A, r)^{2 k_{r}-2 k_{r-1}} t^{2 k_{r}-2 k_{r-1}} p^{-1} \\
& =C_{2}(A, r-1) C_{1}(A, r)^{2 k_{r}-2 k_{r-1}} t^{2 k_{r}}=A \cdot C_{2}(A, r-1)^{2} t^{2 k_{r}}
\end{aligned}
$$

Теперь возьмем $C_{2}(A, r)=A \cdot C_{2}(A, r-1)^{2}+C_{2}(A, r-1)$. Тогда

$$
p^{r} T_{k_{r}}\left(G_{r}\right) \leqslant A \cdot C_{2}(A, r-1)^{2} t^{2 k_{r}}+C_{2}(A, r-1) t^{2 k_{r}}=C_{2}(A, r) t^{2 k_{r}} .
$$

Итак, мы получили (4) при всех $r \geqslant 3$. Поскольку $1 /\left(2 k_{2} k_{r-1}\right)=\delta_{r, n}$, теорема доказана.

ДоказАтельство теоремы 2. Докажем утверждение (ii) (утверждение (i) доказывается аналогично). Имеем

$$
p^{2} T_{n}\left(G_{2}\right)=\sum_{a \in \mathbb{Z}_{p^{*}}^{*}}\left|S\left(a, G_{2}\right)\right|^{2 n}+\sum_{a \in \mathbb{Z}_{p}^{*}}\left|S\left(a, G_{1}\right)\right|^{2 n}+t^{2 n} .
$$

Оценим второе слагаемое:

$$
\sum_{a \in \mathbb{Z}_{p}^{*}}\left|S\left(a, G_{1}\right)\right|^{2 n} \leqslant \sum_{a \in \mathbb{Z}_{p}^{*}}\left|S\left(a, G_{1}\right)\right|^{2} \max _{a \in \mathbb{Z}_{p}^{*}}\left|S\left(a, G_{1}\right)\right|^{2 n-2} \leqslant p t S_{1}^{2 n-2} .
$$

Но неравенство $p t S_{1}^{2 n-2} \leqslant A t^{2 n}$ эквивалентно тому, что

$$
n \geqslant\left\lceil\frac{1}{2} \frac{\ln (p t)-2 \ln S_{1}-\ln A}{\ln t-\ln S_{1}}\right\rceil .
$$

Оценим первое слагаемое:

$$
\sum_{a \in \mathbb{Z}_{p^{*}}^{*}}\left|S\left(a, G_{2}\right)\right|^{2 n} \leqslant \sum_{a \in \mathbb{Z}_{p^{*}}^{*}}\left|S\left(a, G_{2}\right)\right|^{4} \max _{a \in \mathbb{Z}_{p^{2}}^{*}}\left|S\left(a, G_{2}\right)\right|^{2 n-4} \leqslant p^{2} T S_{2}^{2 n-4} .
$$

Видно, что при $n$, определенном формулой (3), выполняется неравенство $p^{2} T S_{2}^{2 n-4} \leqslant$ $A t^{2 n}$. Значит, сумма всех трех слагаемых в (5) не превосходит $(2 A+1) t^{2 n}$.

СледСтвиЕ 2. Пусть выполнено одно из условий:

(i) $S\left(G_{2}\right) \leqslant A p^{\alpha} t^{\beta}<t n p u t \geqslant B p^{\gamma}, \gamma>1 / 2, \gamma-\beta \gamma-\alpha>0$,

$$
n=\max \left(\left\lceil\frac{\gamma}{2 \gamma-1}\right\rceil,\left\lceil 1+\frac{2-\gamma}{2(\gamma-\beta \gamma-\alpha)}\right\rceil\right)
$$

(ii) $T_{2}\left(G_{2}\right) \leqslant A p^{\alpha} t^{\beta}$ npu $t \geqslant B p^{\gamma}, \gamma>1 / 2,4 \gamma-\beta \gamma-\alpha-1>0$,

$$
n=\max \left(\left\lceil\frac{\gamma}{2 \gamma-1}\right\rceil,\left\lceil\frac{2}{4 \gamma-\beta \gamma-\alpha-1}\right\rceil\right) \text {. }
$$


Тогда $T_{n}\left(G_{2}\right) \leqslant C_{3} p^{-2} t^{2 n}$, для некоторой функиии $C_{3}=C_{3}(A, B, \alpha, \beta, \gamma)$.

ДокАзАтельство. В (i) используется оценка $S\left(G_{1}\right) \leqslant \sqrt{p}$, которая получается из основного неравенства при $k=l=1$. Далее можно использовать теорему 2 (или повторить ее доказательство). В (ii) величина $S\left(G_{2}\right)$ оценивается с помощью основного неравенства при $k=l=2$.

ДокАзАТЕЛЬСТво СЛЕДСтвия 1 . Оцениваемая сумма есть просто $S\left(a, G_{2}\right)$ для \# $G_{2}=$ $t=p-1$. Но мы знаем (см. [9]), что $T_{2}\left(G_{2}\right) \leqslant A p^{5 / 2}$. Применяя следствие 2 , (ii) для $A, B=1 / 2, \alpha=5 / 2, \beta=0, \gamma=1$, находим $n=4$. Далее, подставляя найденное $n$ в теорему 1 , получим требуемое неравенство.

Если же $t$ мало, но $t \geqslant p^{\varepsilon}$, то, как следует из работ [11] и [12], найдутся такие $\delta_{1}=$ $\delta_{1}(\varepsilon)>0$ и $\delta_{2}=\delta_{2}(\varepsilon)>0$, что $S\left(G_{1}\right) \leqslant C_{B K}(\varepsilon) t p^{-\delta_{1}}$ и $S\left(G_{2}\right) \leqslant C_{B}(\varepsilon) t p^{-\delta_{2}}$. Применяя теорему 2 , получаем

СлЕДСтвие 3. Пусть $t \geqslant p^{\varepsilon}, \delta_{1}=\delta_{1}(\varepsilon), \delta_{2}=\delta_{2}(\varepsilon)$. Тогда при

$$
n=\max \left(\left\lceil 1+\frac{1-\varepsilon}{2 \delta_{1}}\right\rceil,\left\lceil 1+\frac{2-\varepsilon}{2 \delta_{2}}\right\rceil\right)
$$

будет выполнено неравенство $T_{n}\left(G_{2}\right) \leqslant C_{4}(\varepsilon) p^{-2} t^{2 n}$.

\section{СПИСОК ЦИТИРОВАННОЙ ЛИТЕРАТУРЫ}

[1] И. М. Виноградов, Избраннъе труды, Изд-во АН СССР, М., 1952. [2] И. М. Виноградов, Метод тригонометрических сумм в теории чисел, 2-е изд., Наука, М., 1980. [3] И. М. Виноградов, Особые варианты метода тригонометрических сумм, Наука, М., 1976. [4] А. А. Карацуба, Изв. РАН. Сер. матем., 59:4 (1995), 61-80. [5] А. А. Карацуба, Изв. РАН. Сер. матем., 59:5 (1995), 93-102. [6] J. Friedlander, H. Iwaniec, The BrunTitchmarsh Theorem, Lecture Notes in Statist., 247, Cambridge Univ. Press, Cambridge, 1997. [7] S. Konyagin, I. Shparlinski, Character Sums with Exponential Functions, Cambridge Univ. Press, Cambridge, 1999. [8] С. В. Конягин, IV Международная конферениия "Современные проблемы теории чисел и ее приложения", Актуальные проблемы, Ч. III, Москва, 2002, 86-114. [9] D. R. Heath-Brown, S. V. Konyagin, Quart. J. Math., 51 (2000), 221-235. [10] Ю. В. Малыхин, "Оценки тригонометрических сумм по модулю $p^{2}$ " (в печати). [11] J. Bourgain, S. V. Konyagin, C. R. Math. Acad. Sci. Paris, 337:2 (2003), 75-80. [12] J. Bourgain, Mei-Chu Chang, C. R. Math. Acad. Sci. Paris, 339:7 (2004), 463-466.

Ю. В. Малыхин

Поступило

Московский государственный университет

29.12.2005

им. М. В. Ломоносова

E-mail: jura05@narod.ru 